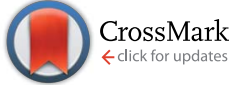

Cite this: RSC Adv., 2016, 6, 32060

\title{
Cytotoxicity of multifunctional surfactant containing capped mesoporous silica nanoparticles $\dagger$
}

\begin{abstract}
Adem Yildirim, *ab $^{\text {Muge Turkaydin, }}{ }^{\mathrm{C}}$ Bora Garipcan ${ }^{\mathrm{C}}$ and Mehmet Bayindir*abd
This paper reports the synthesis of silica capped surfactant (cetyltrimethylammonium bromide; CTAB) and dye (Rose Bengal; RB) containing mesoporous silica nanoparticles (MSNs). Capping the pores of the surfactant containing MSNs with a thin silica layer decreased the immediate surfactant originated cytotoxicity of these particles without affecting their long term ( 3 days) cytotoxicity. Also, the silica capping process almost completely prevented the hemolytic activity of the surfactant containing MSNs. In addition, improved uptake of silica capped MSNs compared to the uncapped particles by cancer cells was demonstrated. The delayed cytotoxicity, low hemolytic activity, and better cellular uptake of the silica capped MSNs make them promising for the development of safe (i.e. with fewer side effects) yet efficient theranostic agents. These nanocarriers may release the loaded cytotoxic molecules (CTAB) mostly after being accumulated in the tumor site and cause so minimal damage to the normal tissues and blood components. In addition, the nanoscale confinement of RB molecules inside the pores of MSNs makes the particles brightly fluorescent. Furthermore, it was demonstrated that due to the singlet oxygen generation capability of the RB dye the silica capped MSNs can be also used for photodynamic therapy of cancer.
\end{abstract}

Received 18th October 2015 Accepted 15th March 2016

DOI: $10.1039 / c 5 r a 21722 a$

www.rsc.org/advances "ultrabright" MSNs with emission intensities comparable to quantum dots have been prepared and used in chemical sensing and bioimaging applications. ${ }^{6,11-15}$ However, high toxicity of surfactant molecules limits the use of ultrabright MSNs in cellular labelling and imaging applications. Therefore, ultrabright MSNs have been used for only short term (a few hours) cell labelling studies; their long-term ( $>4 \mathrm{~h}$ ) effects on cellular viabilities have not been investigated in these studies. In fact, it is well known that even the residual CTAB molecules remaining from an incomplete surfactant extraction process can be very toxic to cells..$^{16,17}$

Some recent studies, ${ }^{7,18-20}$ on the other hand, turn this limitation into a benefit in the therapy of cancer using the high toxicity of CTAB against several cancer cell lines. ${ }^{21-23}$ The lowcost of CTAB and its high weight percentage in MSNs (up to $0.5 \mathrm{~g} \mathrm{~g}^{-1}$ of MSN) make them promising for development of inexpensive nanoscale agents for cancer therapy. In addition, the anti-cancer efficiency of CTAB containing MSNs can be further increased by surfactant assisted loading of other anticancer drugs (e.g. doxorubicin or camptothecin) into the pores of these nanocarriers, which would also be advantageous for the therapy of multidrug resistant cancer cells..$^{19,20}$ The major limitation of this facile method is the instant and non-specific toxicity of CTAB molecules, which are released from the open pores of MSNs, against both normal and cancer cells. To develop an ideal surfactant containing MSN based anticancer nanoagent, release of the СТАВ molecules should be slowed 
down to reduce their immediate toxicity. This may provide enough time to the intravenously injected nanoagents to reach and accumulate in tumor sites without considerably affecting the blood components or healthy tissues. Accordingly, surfactant containing MSNs may show their toxicity mostly on their target tumor sites without damaging the healthy tissues and causing significant side-effects.

Here, we describe the preparation of silica capped and Rose Bengal (RB) dye loaded CTAB containing MSNs to realize a safer surfactant containing anticancer nanoagents (Fig. 1). In addition to its anticancer efficiency, the CTAB surfactant also directs the structure of MSNs and encapsulates the RB molecules into the pores of MSNs. The RB dye provides both fluorescence and singlet oxygen generation ability to the silica capped multifunctional MSNs. The MSNs were capped with a uniform silica layer to slow down the release of CTAB molecules and accordingly reduce their immediate cytotoxicity. Indeed, silica capped MSNs demonstrated initially lower cytotoxicity than uncapped MSNs against both cancer (MCF-7) and non-cancerous (L2929) cells. With increasing incubation times, on the other hand, capped particles demonstrated a similar cytotoxicity profile with uncapped ones. Also, we showed that under illumination of green light, RB loaded capped MSNs can further reduce the cellular viabilities of MCF-7 cells. In addition, silica capping almost completely prevent the hemolytic activity of the surfactant containing MSNs. Furthermore, it was observed that silica capping increases the uptake of the nanoagents by MCF-7 cells.

\section{Results and discussion}

\section{Synthesis and characterization of surfactant containing MSNs}

Synthesis steps of CTAB and RB containing uncapped and silica capped MSNs are outlined in Fig. 1. RB loaded surfactant containing MSNs (named as rMSN) were prepared using the surfactant (CTAB) assistant loading method. To cap the pores of rMSN, excess silica precursor (TEOS) was added after the formation of rMSN in a one-pot reaction, which yields a thin and uniform silica shell around the particles. The silica capped particles were named as rMSN-ts. Also, a surfactant-free MSN (rMSN-ex) was prepared by extracting the CTAB- and accordingly the encapsulated RB- of rMSN for control experiments.

Fig. 2 shows the TEM images of the rMSN and rMSN-ts which have fairly monodisperse particle size distribution with a short rod-like shape. Close up TEM image of rMSN (Fig. 2a, the right column) shows the hexagonally ordered mesostructure of these particles. Formation of a uniform silica shell around $(\sim 8.5 \mathrm{~nm})$ the rMSN-ts can be easily observed from Fig. $2 \mathrm{~b}$. To increase the contrast between core and shell of rMSN-ts and observe the shell more clearly, CTAB inside the pores of rMSN-ts was extracted and their TEM images were taken (Fig. S1, ESI $\dagger$ ), which also shows the silica shell formation around MSNs. In addition, silica capping process did not cause a significant change in the morphology of the particles; hexagonally ordered mesostructured core was still observable. Particle length, width and aspect ratio of the particles were summarized in the Table 1. As expected, length and width of the particles slightly increased after formation of the silica shell. Also, aspect ratio was slightly decreased after silica shell formation due to the uniform size increase in both directions.

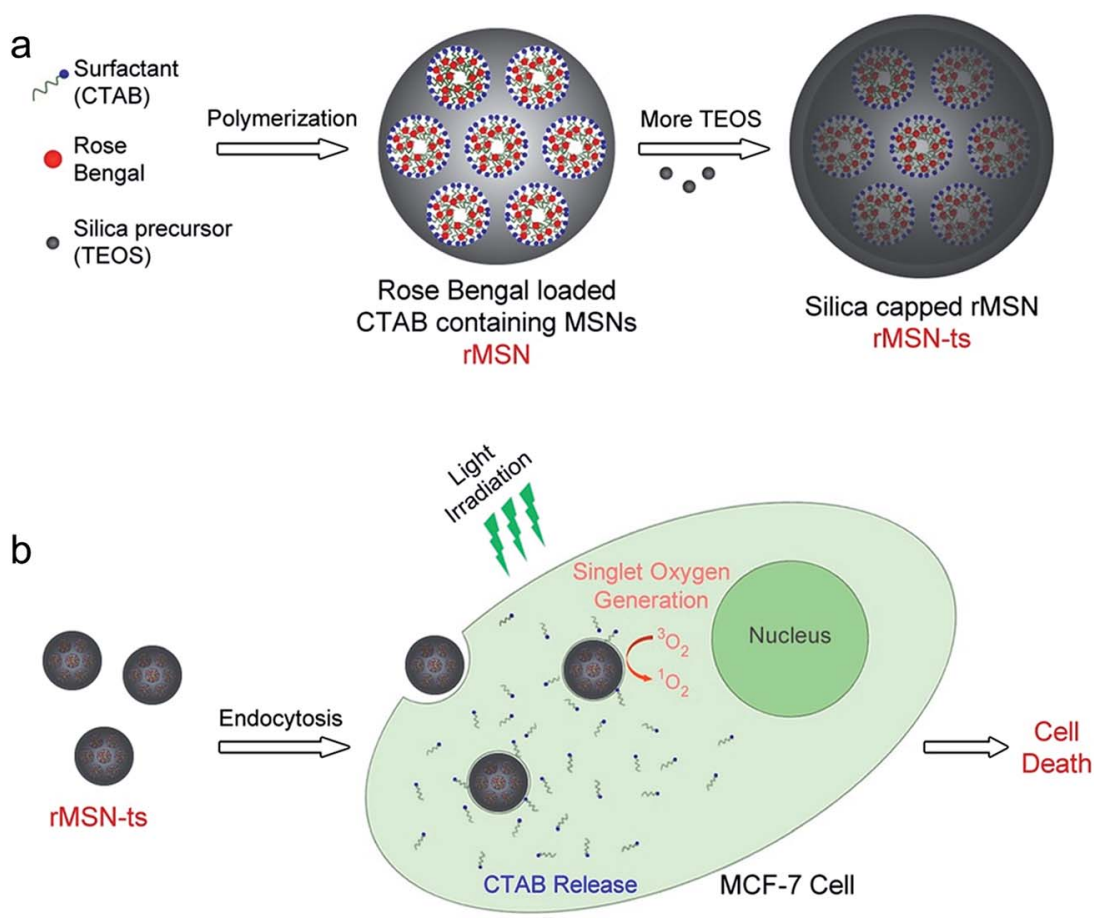

Fig. 1 Schematic representation of (a) silica capped MSN synthesis and (b) proposed cancer cell killing mechanism by silica capped MSNs. 
a
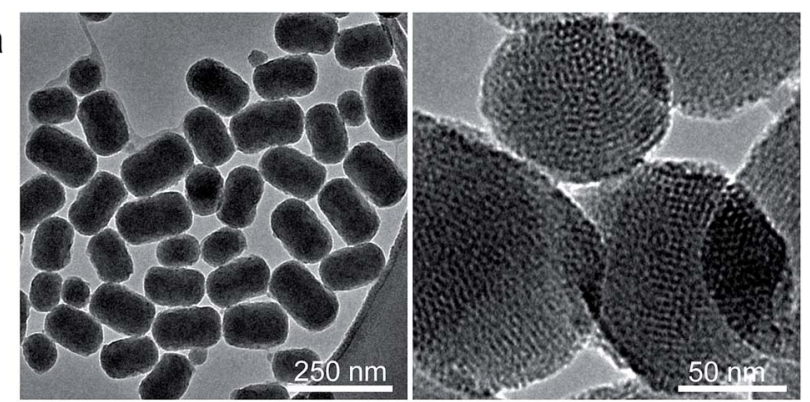

b

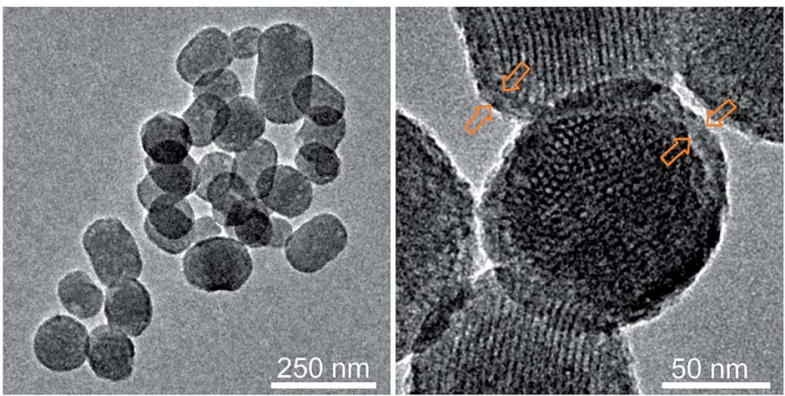

Fig. 2 TEM images of the surfactant containing MSNs; (a) rMSN and (b) rMSN-ts. Left and right panels show the low and high magnification images of the particles, respectively. Both particles have short rod-like shape and fairly monodisperse in size. Uniform silica layer around rMSN-ts can be easily observed from the images. Orange arrows in (b) indicate the nonporous shells formed around the mesoporous cores of rMSN-ts.

Surfactant contents of the nanocarriers were determined based on their TGA curves (Fig. 3). The weight loss for rMSN-ex was $9.9 \%$ at around $800{ }^{\circ} \mathrm{C}$ which is due to the decomposition of methyl and propyl phosphonate groups (added to make the pores of particles hydrophobic ${ }^{15}$ and increase the water dispersibility ${ }^{24}$ of the particles, respectively) and dehydroxylation of the silica surface. ${ }^{25}$ As expected, the weight losses were higher for rMSN and rMSN-ts; $44 \%$ and $29 \%$, respectively due to the presence of $\mathrm{CTAB}$ and $\mathrm{RB}$ molecules inside their pores. Accordingly, CTAB contents were calculated to be $354.7 \mathrm{mg} \mathrm{g}^{-1}$ for rMSN and $197.3 \mathrm{mg} \mathrm{g}^{-1}$ for rMSN-ts (Table 1). The high surfactant content of MSNs make them promising for efficient therapy of cancer. RB contents of the nanocarriers were determined by extracting the loaded RB from the pores using ethanol which can penetrate into the hydrophobic pores and dissolve the encapsulated RB molecules. Particles dispersed in ethanol were precipitated by centrifugation, supernatants were collected and extracted RB was detected by using a UV-vis spectrophotometer. This treatment was repeated until there was no detectable RB in the supernatants. The RB contents of the

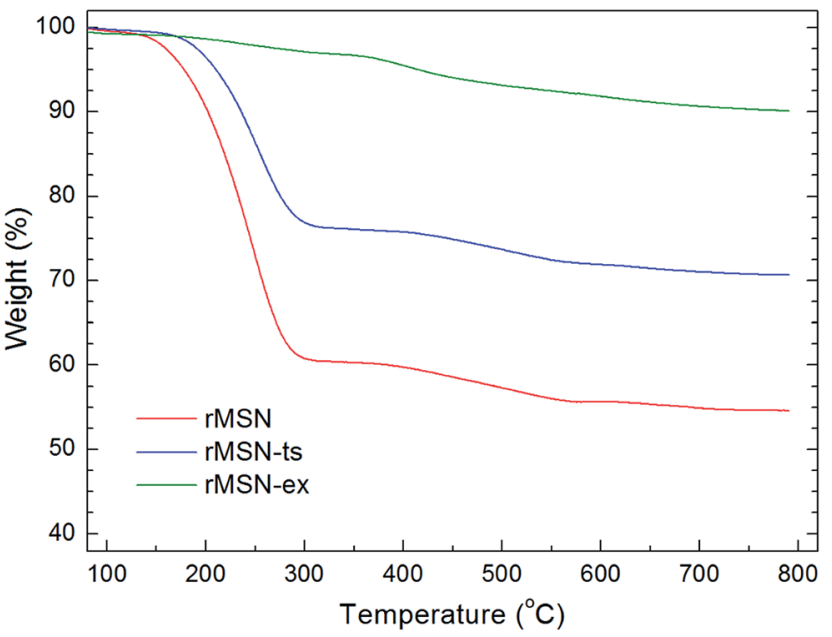

Fig. 3 TGA curves of the rMSN, rMSN-ts and rMSN-ex.

nanocarriers were calculated to be $8.6 \mathrm{mg} \mathrm{g}^{-1}$ and $5.1 \mathrm{mg} \mathrm{g}^{-1}$ for rMSN and rMSN-ts, respectively (Table 1). Both CTAB and RB amounts in per gram of nanocarrier were lower for rMSN-ts, due to the surfactant and dye free silica shells of these nanocarriers.

The zeta potentials of MSNs dispersed in PBS $(10 \mathrm{mM}, \mathrm{pH}$ 7.4) were measured in order to characterize their surface properties (Table 1). The net surface charge of rMSN was slightly positive $(3.3 \mathrm{mV})$ as a result of the presence of both positively charged CTAB molecules in the pore openings and negatively charged silanol and phosphonate groups at the silica surface. Apparently, CTAB was more abundant on the rMSN surface compared to the negatively charged surface groups. After surfactant extraction (rMSN-ex), surface became negatively charged $(-6.3 \mathrm{mV})$ due to the removal of CTAB molecules. On the other hand, surface charge of rMSN-ts was highly negative $(-13.1 \mathrm{mV})$ due to the existence of only negatively charged silanol and phosphonate groups on the silica surface; CTAB molecules were not exposed to the interface since they are under the silica shell. Also, nonporous surface of rMSN-ts is more negatively charged than porous surface of rMSN-ex, which in accordance with the previous reports. ${ }^{26}$

Optical properties of the rMSN and rMSN-ts were studied and compared with free RB molecules at same dye concentration $\left(5 \mu \mathrm{g} \mathrm{mL} \mathrm{m}^{-1}\right)$. Fig. 4 a reveals that the absorption maxima of RB molecules confined in the pores of rMSN and rMSN-ts were approximately $10 \mathrm{~nm}$ red shifted compared to the free RB molecules, which is due to the nonpolar environment inside the nanochannels. ${ }^{27}$ Similarly, we observed a red shift of around 15 $\mathrm{nm}$ in the fluorescence spectra of both rMSN and rMSN-ts

Table 1 Physical properties of the mesoporous silica nanoparticles

\begin{tabular}{|c|c|c|c|c|c|c|c|c|}
\hline Sample & $\begin{array}{l}\text { CTAB } \\
\text { exchange }\end{array}$ & $\begin{array}{l}\text { Aspect } \\
\text { ratio }\end{array}$ & $\begin{array}{l}\text { Length } \\
(\mathrm{nm})\end{array}$ & $\begin{array}{l}\text { Width } \\
\text { (nm) }\end{array}$ & $\begin{array}{l}\text { Shell thickness } \\
(\mathrm{nm})\end{array}$ & $\begin{array}{l}\text { RB content } \\
\left(\mathrm{mg} \mathrm{g}^{-1}\right)\end{array}$ & $\begin{array}{l}\text { CTAB content } \\
\left(\mathrm{mg} \mathrm{g}^{-1}\right)\end{array}$ & $\begin{array}{l}\text { Zeta potential } \\
\text { in PBS }(\mathrm{mV})\end{array}$ \\
\hline rMSN & No & 1.38 & $119 \pm 30$ & $86 \pm 14$ & N/A & 8.6 & 354.7 & $3.3 \pm 0.9$ \\
\hline rMSN-ex & Yes & 1.38 & $119 \pm 30$ & $86 \pm 14$ & N/A & N/A & N/A & $-6.3 \pm 0.3$ \\
\hline
\end{tabular}



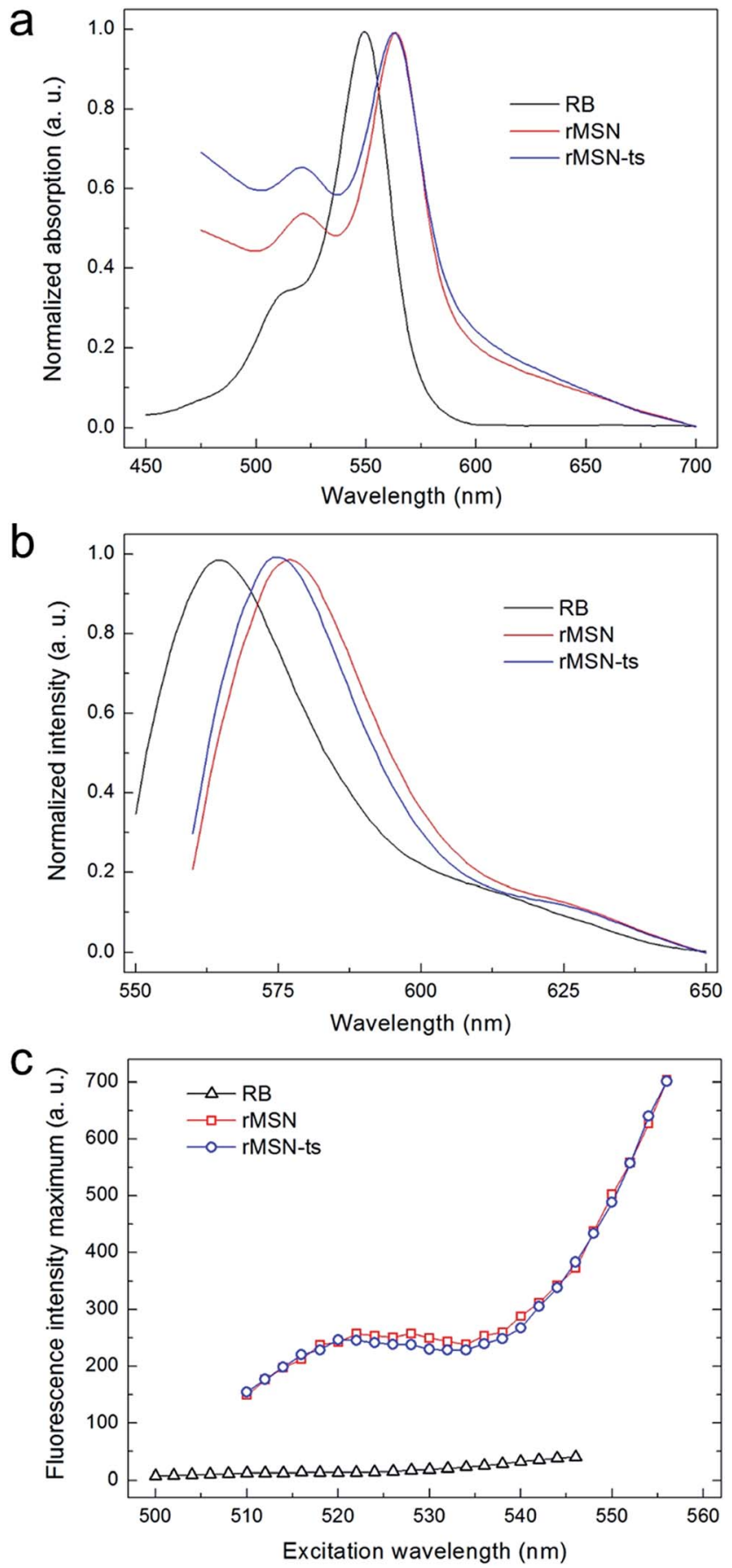

Fig. 4 (a) Normalized UV-vis spectra of free RB molecules and RB loaded MSNs, (b) normalized fluorescence spectra of free RB molecules and RB loaded MSNs, (c) fluorescence maxima of free RB molecules and RB loaded MSNs at different excitation wavelength.

(Fig. 4b). To explore the brightness of particles, we collected the fluorescence spectra of particles and free RB molecules at same dye concentration $\left(5 \mu \mathrm{g} \mathrm{mL} \mathrm{mL}^{-1}\right)$ and at various excitation wavelengths. Fig. 4c clearly shows that when the RB molecules are confined in the pores of MSNs their fluorescence enhanced up to 20 fold depending on the excitation wavelength. Note that, both rMSN and rMSN-ts demonstrated almost the same fluorescence intensity at all excitation wavelengths indicating that the silica capping process has no effect on the fluorescence of the particles.

\section{Cytotoxicity of surfactant containing MSNs}

Cytotoxic effects of surfactant containing MSNs were studied using two different cell lines; as non-cancerous and cancer cells, murine fibroblast L2929 and MCF-7 human breast cancer cell lines were used, respectively. Fig. 5 shows the 3(4,5-dimethylthiazol-2-yl)-2,5-diphenyl-tetrazolium bromide (MTT) assay results for both cell lines after short $(4 \mathrm{~h})$, moderate (24 h) and long (72 h) incubation with rMSN, rMSNts and rMSN-ex in the particle concentration range of 10 to 200 $\mu \mathrm{g} \mathrm{mL}^{-1}$. Uncapped rMSN showed immediate toxicity against both cell lines; even after $4 \mathrm{~h}$ of incubation, cell viabilities were significantly reduced. This extreme toxicity of rMSN is due to the release of toxic CTAB surfactant from the open pores of this nanocarriers. ${ }^{7}$ On the other hand, a delayed toxicity profile was observed for rMSN-ts on both cell lines thanks to the slow release of CTAB molecules from silica capped pores. After $4 \mathrm{~h}$, no significant cytotoxicity was observed even at the highest studied particle concentration of $200 \mu \mathrm{g} \mathrm{mL}^{-1}$. After a $24 \mathrm{~h}$ of incubation, rMSN-ts decreased the viability of both cell lines especially at high particle concentrations. Yet cellular viabilities are significantly higher for rMSN-ts than rMSN for all cases. After $72 \mathrm{~h}$, both rMSN and rMSN-ts largely reduced the cell viabilities around $10 \%$ for both cell lines. To demonstrate the slow cargo release from silica capped particles, we studied RB release from rMSN and rMSN-ts (Fig. 6). In fact, the release of RB from rMSN was almost 6 fold higher than its release from rMSN-ts after 3 $\mathrm{d}$ incubation in PBS, which suggests that thin silica layer can slow down the CTAB release. Note that another reason of lowered toxicity of rMSN-ts is its lower CTAB percentage. Therefore, we also calculated the half maximal inhibitory concentration $\left(\mathrm{IC}_{50}\right.$ ) values (where applicable) of the MSNs based on the CTAB concentration (Table S1, see the ESI†). We observed that $\mathrm{IC}_{50}$ values of rMSN-ts were significantly higher than rMSN for most of the cases which also proves the lower cytotoxicity of the capped nanocarriers. We also studied the effect of surfactant free rMSN-ex on cell viability as negative control of CTAB induced cytotoxicity. We observed that rMSNex decreased the cellular viability at only high particle concentrations after especially $72 \mathrm{~h}$ of incubation. While MSNs are generally considered as non-toxic to the cells, they can show toxicity against cells depending on several factors such as particle size, shape, porosity, surface chemistry, cell type, and incubation time. ${ }^{28}$ In addition, the surfactant extraction method and its efficiency are important since remaining surfactant molecules can also cause cytotoxicity. ${ }^{\mathbf{1 6}}$ Therefore, we believe that the observed cytotoxicity of rMSN-ex at extended incubation times is due to its inherent toxicity (i.e. toxicity originated from its physical and chemical properties) and/or remaining CTAB molecules. This result also indicates that the cytotoxicity of surfactant containing silica nanocarriers mostly originated from the CTAB molecules present in 

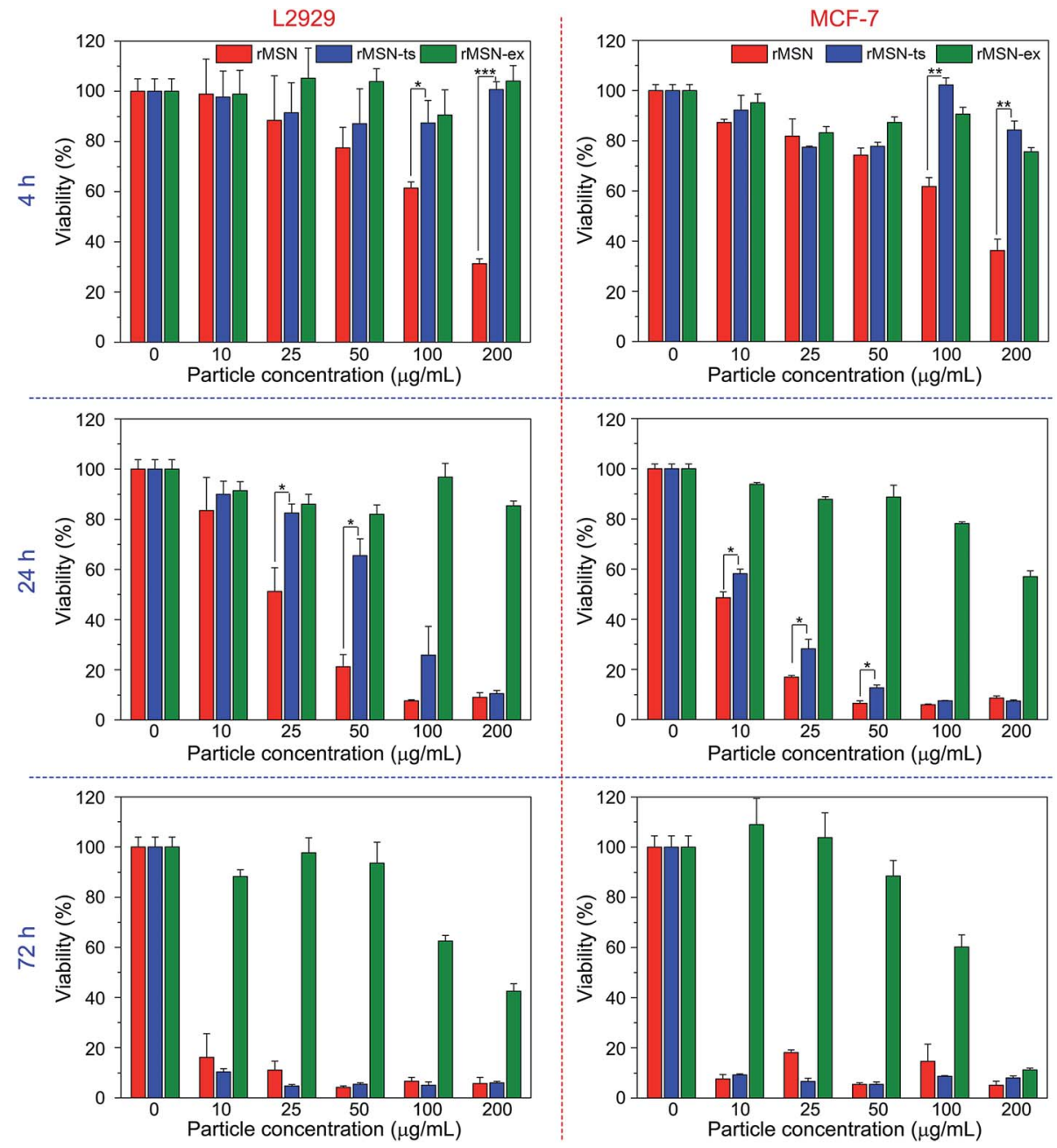

Fig. 5 Cytotoxicity results of surfactant containing MSNs against non-cancerous (L2929) and cancer (MCF-7) cell lines. In general, silica capped MSNs (rMSN-ts) demonstrated lower cytotoxicity than uncapped ones ( $r M S N$ ) especially after $4 \mathrm{~h}$ and $24 \mathrm{~h}$ of incubation. On the other hand, after $72 \mathrm{~h}$ of incubation both rMSN and rMSN-ts demonstrated high cytotoxicity against both cell lines. Lastly, surfactant extracted particles (rMSN-ex) demonstrated cytotoxicity only after $72 \mathrm{~h}$ of incubation at high particle concentrations. Error bars are standard error of the mean. $*$ indicates $p<$ $0.05, * *$ indicates $p<0.001$, and $* * *$ indicates $p<0.0001$ according to the student's $t$-test.

the pores. In addition, it should be noted that, the toxicity of CTAB containing MSNs against MCF-7 cells and L2929 cells were similar, which is consistent with previous reports. ${ }^{16,29}$

\section{Hemolytic activity of surfactant containing MSNs}

A hemolysis assay was used to determine the toxicity of CTAB containing MSNs on red blood cells (RBCs). RBCs were treated with MSNs at the concentration range of 10 to $200 \mu \mathrm{g} \mathrm{mL}{ }^{-1}$, for 2 hours. Fig. 7a shows the concentration dependent hemolytic activity of the MSNs. rMSN demonstrated a high hemolytic activity; at $200 \mu \mathrm{g} \mathrm{mL} \mathrm{m}^{-1} 35.8 \%$ and at $100 \mu \mathrm{g} \mathrm{mL} \mathrm{m}^{-1} 7.1 \%$ of RBCs were hemolyzed. Capping the particles with a thin silica layer drastically decreased the hemolytic activity; rMSN-ts caused hemolytic activities of only $3.1 \%$ and $1.4 \%$ at 200 and $100 \mu \mathrm{g}$ $\mathrm{mL}^{-1}$, respectively. In addition, hemolytic activity of the surfactant extracted particles were investigated; rMSN-ex did not reveal any detectable hemolysis in the studied concentration region due to the phosphonate functional group on its surface. ${ }^{30}$

In order to further investigate the origin of the hemolytic activity of rMSN, we also incubated the RBCs with the supernatants of rMSN and rMSN-ts which are incubated in PBS for $2 \mathrm{~h}$ before administration (Fig. 7b). It is well known that released surfactant molecules (CTAB) can cause lysis of RBCs. ${ }^{31}$ However, we did not observe any remarkable supernatant originated hemolytic activity at either low $\left(25 \mu \mathrm{g} \mathrm{mL}^{-1}\right)$ or high $(200 \mu \mathrm{g}$ $\mathrm{mL}^{-1}$ ) particle concentrations. These results revealed that the hemolytic activity of the surfactant containing MSNs is mainly related with the surface immobilized CTAB molecules which has a positive charge and may strongly interact with the RBC membrane. ${ }^{32}$ Capping the particles with a thin silica layer significantly reduce the hemolytic activity of surfactant containing MSNs by preventing the interactions between positively charged CTAB surfactant molecules and RBC membrane. 


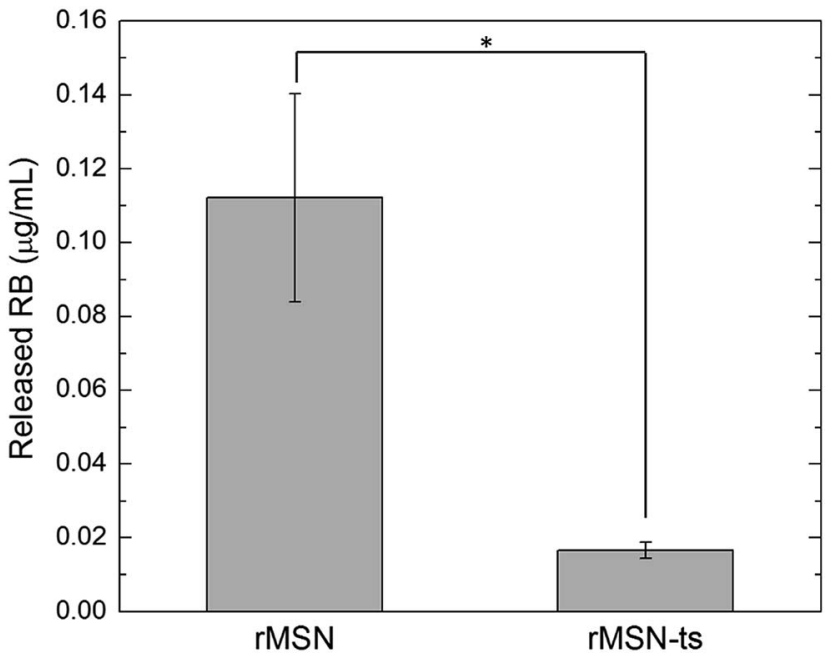

Fig. 6 Rose Bengal release from rMSN and rMSN-ts after incubating the particles $\left(6 \mathrm{mg} \mathrm{mL}^{-1}\right)$ in PBS for $3 \mathrm{~d}$. Error bars are standard deviation. * indicates $p<0.05$ according to the student's $t$-test.

\section{Cellular uptake of surfactant containing MSNs}

Fig. 8 shows the fluorescence and bright field optical microscope images of the MCF-7 cells that were incubated with rMSN and rMSN-ts (particle concentration was $100 \mu \mathrm{g} \mathrm{mL}{ }^{-1}$ ) for $4 \mathrm{~h}$. Less fluorescence was observed for rMSN compared to the rMSN-ts, indicating that rMSN-ts was more effectively uptaken by MCF-7 cells. To further show that rMSN-ts was uptaken more by MCF-7 cells, we calculated the fluorescence intensities per cell using ImageJ from separate images and by counting at least 250 cells (Fig. 8b). The fluorescence intensity per cell was almost 4 fold higher for rMSN-ts than rMSN. Also, the fluorescence signal of rMSN mainly concentrated on the edges of the cells showing that most of the positively charged rMSNs attached to the cell membrane instead of being uptaken by the cells (Fig. 8a). On the other hand, fluorescence signal homogeneously distributed inside the cells for rMSN-ts indicating that the particles were uptaken and dispersed in the cytoplasm of the cells. In general, charged nanoparticles were uptaken better than uncharged nanoparticles, which is due to the electrostatic interactions between charged groups on the nanoparticle surface with the zwitterionic cell membrane. When positive and negative charged compared, it has been generally observed that positively charged nanoparticles were uptaken better by cells than their negatively charged counterparts since the cell membrane is negatively charged. However, several exceptions have been also reported in the literature. ${ }^{33}$ Other factors such as particle aggregation, surface hydrophobicity and absolute value of surface charge may also affect the cellular uptake of nanoparticles. In fact, absolute value of zeta potential of rMSN-ts is much larger than rMSN, which are 13.1 and 3.3, respectively. Therefore, it is believed that better uptake of rMSN-ts is due to its higher surface charge.

In addition, many dead (spherical) cells can be observed from the low magnification bright-field images for rMSN treated cells (Fig. S2, see the ESI $\dagger$ ). On the other hand, the morphology of rMSN-ts treated cells was similar with the control, which indicates most of the cells were alive. This observation was in accordance with the MTT results indicating that silica capping significantly reduced the immediate toxicity of surfactant containing MSNs.

\section{Phototoxicity of surfactant containing MSNs}

In addition to their chemotoxicity, rMSN and rMSN-ts are expected to be phototoxic to the cells due to the encapsulated $\mathrm{RB}$ molecules, which is a well-known photosensitizer (i.e. produces singlet oxygen when illuminated with light at suitable wavelengths). ${ }^{34,35}$ The generation of singlet oxygen in the presence of rMSN and rMSN-ts was demonstrated by photobleaching of the 1,3-diphenylisobenzofuran (DPBF) dye (Fig. S3, see the ESI $\dagger$ ). ${ }^{36}$ It was observed that after silica capping, singlet oxygen generation capability of rMSN was partially quenched, most likely due to the silica shell barrier between dye and the oxygen molecules. In a proof of concept demonstration, we investigated the phototoxicity of rMSN and rMSN-ts using MCF-7 cells (Fig. 9).
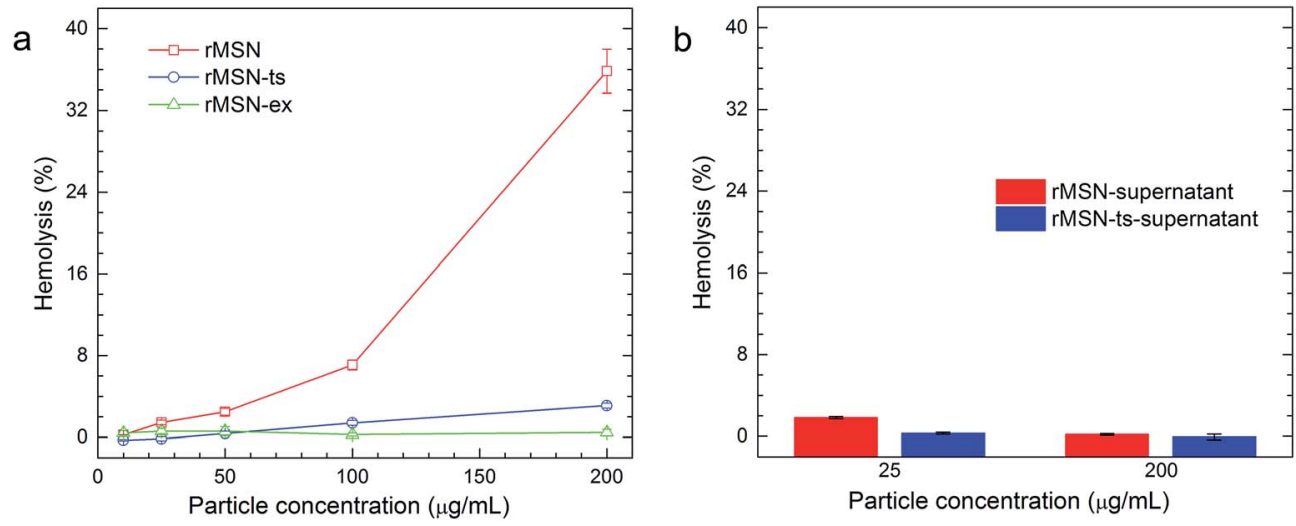

Fig. 7 (a) Hemolytic activity of rMSN, rMSN-ts and rMSN-ex. (b) Hemolytic activity of supernatants of rMSN and rMSN-ts. Capping the particles with a thin silica shell significantly reduced their hemolytic activity. Error bars are standard deviation. Student's $t$-test analyses revealed the statistical significance of the data $(p<0.05)$. 
a
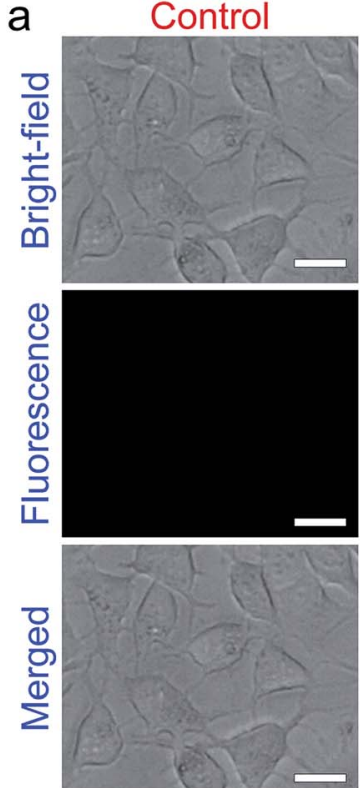

rMSN
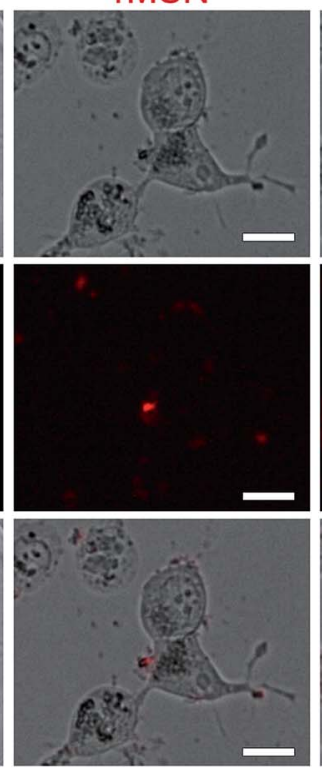

rMSN-ts

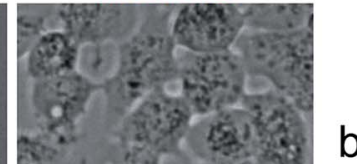

b

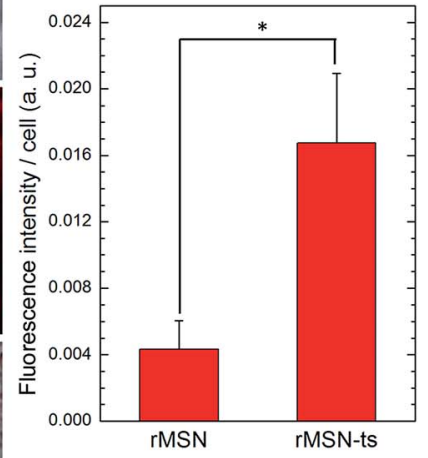

Fig. 8 (a) Fluorescence, bright field and merged images of MCF-7 cells which were untreated or treated with $100 \mathrm{mg} \mathrm{mL}^{-1}$ of CTAB containing particles for $4 \mathrm{~h}$. Cellular uptake of rMSN-ts was significantly higher than that of rMSN indicating that silica capping promotes the uptake of surfactant containing MSNs and as well as reduce the cytotoxicity. (b) Fluorescence intensity of MCF-7 cells which were untreated or treated with $100 \mathrm{mg} \mathrm{mL}^{-1}$ of CTAB containing particles for $4 \mathrm{~h}$. Error bars are standard deviation. ${ }^{*}$ indicates $p$.

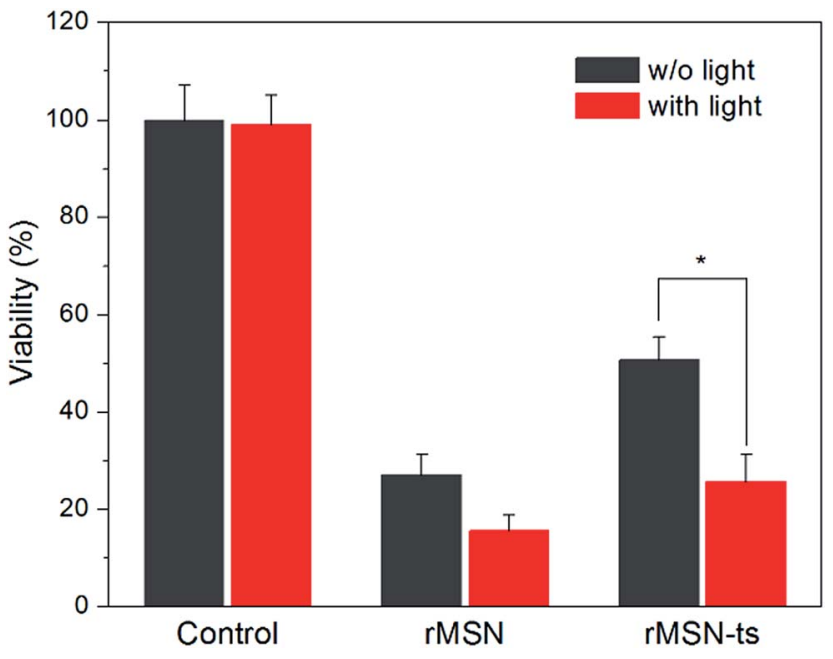

Fig. 9 Photodynamic therapy results of MCF-7 cells. Viability of the cells treated with $100 \mu \mathrm{g} \mathrm{mL}^{-1}$ of particles for $4 \mathrm{~h}$ after illumination or no illumination with a green laser for $30 \mathrm{~s}$. Error bars are standard error of the mean. * indicates $p<0.05$ according to the student's $t$-test.

First cells were incubated with particles for $4 \mathrm{~h}$ at the particle concentration of $100 \mu \mathrm{g} \mathrm{mL}{ }^{-1}$ and then the particle containing medium was removed and cells were further incubated for $16 \mathrm{~h}$ in a particle-free medium. After incubation, half of the cells were illuminated using a green laser $(5 \mathrm{mV})$ for $30 \mathrm{~s}$ and the other half was not. In the absence of light, both rMSN and rMSN-ts reduced the viability of MCF-7 cells due to their chemotoxicity. Also, as expected more reduction in cell viability was observed for rMSN. In the presence of light, the cell viabilities were further reduced to around $20 \%$ for both rMSN and rMSNts. Although RB content and singlet oxygen generation capability of rMSN-ts are lower than those of rMSN, the photoinduced reduction in the viability was more pronounced for rMSN-ts, which may be due to its higher uptake capacity by MCF-7 cells.

\section{Conclusions}

In summary, silica capped and dye doped surfactant containing brightly fluorescent MSNs were prepared and their cytotoxicity, haemolytic activity and cellular uptake was studied. MSNs were doped with the photosensitizer and fluorescent RB dye using the surfactant-assisted loading method and capped with a uniform silica shell in a one-pot reaction. It was observed that silica capping decrease the immediate toxicity of surfactant containing MSNs against both L2929 and MCF-7 cell lines. Also, hemolysis can be induced by the particles was investigated using RBCs. In addition, cellular uptake of particles and their potential in PDT were studied using by MCF-7 cells.

When these nanocarriers are intravenously injected, their accumulation in the tumor region can be expected through EPR effect due to their small size. ${ }^{37}$ In addition, targeting ligands (such as folic acid, transferrin, RGD peptide etc.) can be attached to their surface to improve their localization in the tumor region. ${ }^{38}$ Small particle size, with suitable surface chemistry, can also provide them long blood circulation time by escaping the reticular endothelial system (RES). ${ }^{39}$ While rapid CTAB release can be expected for uncapped MSNs after entering the blood, CTAB release should be much slower for our silica capped MSNs. In fact, around 6 fold reduction in the release was 
observed after capping the particles with a thin silica shell (Fig. 6). Therefore, reducing the CTAB release rate may reduce the possible side-effects of the particles by giving enough time to the particles to accumulate in the target region. In addition, drug (CTAB) content of the nanocarriers is very high; therefore slowing down the release may provide more sustained release of the drug and long term anti-cancer efficiency for the particles, which are reached their targets. Note that, other cargo molecules such as dyes and drugs can be easily added to the particles using surfactant assistant loading method to give the particles other functionalities. Moreover, other advantages of silica capping were also observed; (i) silica capping significantly reduces the hemolytic activity of the nanocarriers and (ii) improves the cellular uptake. We believe that the preliminary results obtained in this study show the promising potential of capped surfactant containing multifunctional MSNs in biomedical applications. Future studies may include surface modification of silica capped surfactant containing MSNs with antifouling polymers and targeting agents and their in vivo verification.

\section{Experimental section}

\section{Materials}

Cetyltrimethylammonium bromide (CTAB), F127 pluronic polymer, 3-(trihydroxysilyl)propyl methylphosphonate (TSPMP), Rose Bengal, 3-(4,5-dimethyl-2-thiazolyl)-2,5diphenyltetrazolium bromide (MTT), and 1,3-diphenylisobenzofuran were purchased from Sigma Aldrich (USA). Tetraethyl orthosilicate (TEOS) and methyltrimethoxysilane (MTMS) were purchased from Merck (Germany). All chemicals were used as received.

\section{Preparation of surfactant containing MSNs}

MSNs were prepared by modifying previous reports. ${ }^{15,40-42}$ To synthesize rMSN, $200 \mathrm{mg}$ CTAB and $10 \mathrm{mg}$ of F127 were dissolved in $95 \mathrm{~mL}$ of water and $0.8 \mathrm{~mL}$ of $\mathrm{RB}$ solution in water $(5$ $\left.\mathrm{mg} \mathrm{mL}{ }^{-1}\right)$ and $0.7 \mathrm{~mL}$ of $\mathrm{NaOH}(2 \mathrm{M})$ were added to this solution. Then the reaction mixture was heated to $80^{\circ} \mathrm{C}$ while stirring vigorously $(600 \mathrm{rpm})$. After temperature fixed at $80^{\circ} \mathrm{C}, 1 \mathrm{~mL}$ of TEOS was rapidly added to the reaction mixture. After $15 \mathrm{~min}$, $60 \mu \mathrm{L}$ of MTMS and after $45 \mathrm{~min}, 250 \mu \mathrm{L}$ of TSPMP was added dropwise and reaction mixture was further stirred for $90 \mathrm{~min}$. Finally, particles were collected by centrifugation at $9000 \mathrm{rpm}$ for $20 \mathrm{~min}$ and washed with water twice. To synthesize rMSN-ts, $1 \mathrm{~mL}$ of TEOS was added dropwise to the reaction mixture 20 min before the TSPMP addition. Other parameters were same with the rMSN synthesis. To prepare rMSN-ex, surfactant and $\mathrm{RB}$ molecules of rMSN were extracted by dispersing the particles in $50 \mathrm{~mL}$ of $20 \mathrm{~g} \mathrm{~L}^{-1}$ ethanolic ammonium nitrate solution and stirred vigorously at $60{ }^{\circ} \mathrm{C}$ for $30 \mathrm{~min} .{ }^{43}$ This treatment was repeated twice. Then the particles were washed with water twice and dried at $60{ }^{\circ} \mathrm{C}$.

\section{Cell culture}

Human breast adenocarcinoma cells (MCF-7) and murine fibroblast cells (L2929) were grown to confluence at $37{ }^{\circ} \mathrm{C}$ under $5 \% \mathrm{CO}_{2}$ in Dulbecco's Modified Eagle Serum (DMEM) containing $1 \%$ penicillin/streptomycin, $10 \%$ fetal bovine serum (FBS) and $2 \mathrm{mM}$ L-glutamine.

\section{Cell viability tests}

The viability of MCF-7 and L929 cells, treated with MSNs, were tested by MTT assay. $10^{4}$ cells per well were seeded to 96-well plates for both cell lines. After overnight incubation, MSNs in 11 $\mu \mathrm{L}$ of PBS were added to each well to give a final nanoparticle concentration between 10 and $200 \mu \mathrm{g} \mathrm{mL}^{-1}$. Then the cells were incubated for 4,24 or $72 \mathrm{~h}$. The cytotoxicity of particles was determined by the MTT reduction assay. First MSN containing medium was removed and $90 \mu \mathrm{L}$ of fresh medium was gently added. Then $20 \mu \mathrm{L}$ of filter-sterilized MTT reagent $\left(5 \mathrm{mg} \mathrm{mL}^{-1}\right.$ in PBS) was added to each well, and the plates were incubated at $37{ }^{\circ} \mathrm{C}$ for $4 \mathrm{~h}$. After incubation, medium was removed and the precipitated formazan crystals were dissolved by adding acidic isopropyl alcohol. Optical density of dissolved formazan crystals was measured at 570 and $655 \mathrm{~nm}$ using a microplate reader (iMark, Biorad). The untreated cells were considered as reference for the calculations. ${ }^{\mathbf{4 4}}$ All the samples were prepared in triplicate. Student's $t$-test was applied to all datasets and the difference between them was accepted to be statistically significant when $p<0.05$.

\section{Fluorescence microscopy}

$5 \times 10^{4}$ MCF-7 cells per well were seeded to 24 -well plates and cultured for $48 \mathrm{~h}$. After culture, MSNs in $55 \mu \mathrm{L}$ of PBS were added to the wells to give final nanoparticle concentrations of $100 \mu \mathrm{g} \mathrm{mL}^{-1}$. After $4 \mathrm{~h}$ of incubation, nanoparticle containing mediums were removed, cells were washed with PBS three times. Finally, optical and fluorescence images of cells were taken in PBS using a fluorescence microscope (DMIL, Leica) using a $40 \times$ lens and green and red filters for excitation and emission, respectively.

\section{Rose Bengal release studies}

To determine the amount of released RB from the capped and uncapped MSNs, rMSN or rMSN-ts was dispersed in PBS (10 $\mathrm{mM}, \mathrm{pH}$ 7.4) at a particle concentration of $6 \mathrm{mg} \mathrm{mL}^{-1}$. Then, dispersions were stirred at $37{ }^{\circ} \mathrm{C}$ for $3 \mathrm{~d}$. Finally, MSNs were precipitated by centrifugation and supernatants were collected. The released $\mathrm{RB}$ amounts were determined by recording the absorption of released RB at $550 \mathrm{~nm}$ using an UV-vis spectrophotometer (Cary 100, Varian). All the samples were prepared in triplicate. Student's $t$-test was applied to all datasets and the difference between them was accepted to be statistically significant when $p<0.05$.

\section{PDT experiments}

$10^{4}$ MCF-7 cells per well were seeded to 96-well plates and cultured for $48 \mathrm{~h}$. After culture, nanoparticles in $11 \mu \mathrm{L}$ of PBS 
were added to the wells to give final nanoparticle concentrations of $100 \mu \mathrm{g} \mathrm{mL} \mathrm{m}^{-1}$. After $4 \mathrm{~h}$ of incubation, nanoparticle containing mediums were removed, cells were washed with PBS, fresh medium was added and cells were further incubated for $16 \mathrm{~h}$. Then, cells were illuminated with a $5 \mathrm{mV}$ green laser for $30 \mathrm{~s}$ and incubated for $1 \mathrm{~h}$. Finally, cell viabilities were determined using a MTT assay. ${ }^{44}$ All the samples were prepared in triplicate. Student's $t$-test was applied to all datasets and the difference between them was accepted to be statistically significant when $p<0.05$.

\section{Hemolysis assay}

Hemolysis experiments were performed according to a previous report. ${ }^{31}$ EDTA stabilized human blood samples were freshly obtained from volunteers at Bilkent University Health Center (Ankara, Turkey). All experiments were performed in compliance with the relevant laws and institutional guidelines. The experimental methods were approved by the ethics committee of Bilkent University and also informed consents were obtained from the volunteers. First, $2 \mathrm{~mL}$ of blood was centrifuged at $1600 \mathrm{rpm}$ for $5 \mathrm{~min}$ and blood plasma and surface layer was removed. Remaining RBC pellet was washed five times with $5 \mathrm{~mL}$ of PBS solution and RBCs were diluted in $20 \mathrm{~mL}$ of PBS solution. Then, 0.8 $\mathrm{mL}$ of nanoparticle solutions or supernatants in PBS at different concentrations was added to $0.2 \mathrm{~mL}$ of $\mathrm{RBC}$ suspension. Also, positive and negative control samples were prepared by adding $0.8 \mathrm{~mL}$ of water and PBS, respectively to $0.2 \mathrm{~mL}$ of RBC solution. Then, samples were incubated at 25 ${ }^{\circ} \mathrm{C}$ for $2 \mathrm{~h}$. The samples were slightly shaken once for every 30 min to resuspend the RBCs and MSNs. After $2 \mathrm{~h}$, samples were centrifuged at $1600 \mathrm{rpm}$ and $150 \mu \mathrm{L}$ of supernatants was transferred to a 96-well plate. Absorbance of hemoglobin in supernatants was measured with a microplate reader at 570 $\mathrm{nm}$. Also, absorbance at $655 \mathrm{~nm}$ was recorded as reference. Percent hemolysis values were calculated from three separate experiments. Student's $t$-test was applied to all datasets and the difference between them was accepted to be statistically significant when $p<0.05$.

\section{Characterization}

Transmission electron microscopy (TEM) images were taken using a Tecnai G2 F30 (FEI) microscope. Thermal gravimetric analyses (TGA) were performed with Q500, (TA Instruments). Zeta potentials of nanoparticles were measured with Zetasizer Nanoseries (Malvern Instruments). Optical absorption measurements in cytotoxicity and hemolysis assays were carried out using Microplate readers (Spectramax M5, Molecular Devices and iMark, Biorad). Absorption spectra of MSNs were recorded using a UV-vis absorption (Cary 100, Varian) spectrophotometer. Fluorescence spectra of the nanoparticles were recorded by a Fluorescence spectrophotometer (Eclipse, Varian).

\section{Acknowledgements}

We would like to thank Assoc. Prof. Dr Nesrin Özeren, Boğaziçi University, Department of Molecular Biology and Genetics, for kindly supplying of L929 fibroblast and MCF-7 breast cancer cell lines and Fahri Emre Öztürk for fruitful discussions. This work is supported by TÜBİTAK under Project No. 111T696. Also, this work was partially supported by Boğaziçi University Research Fund with Grant Number: 6701, 7567. A. Y. is supported by a TÜBITTAK-BIDEB Ph.D. fellowship. M. B. acknowledges partial support from the Turkish Academy of Sciences (TÜBA).

\section{References}

1 I. Sokolov and D. O. Volkov, J. Mater. Chem., 2010, 20, 42474250.

2 H. M. Chen, J. H. He, H. M. Tang and C. X. Yan, Chem. Mater., 2008, 20, 5894-5900.

3 C. H. Tsai, J. L. Vivero-Escoto, I. I. Slowing, I. J. Fang, B. G. Trewyn and V. S. Y. Lin, Biomaterials, 2011, 32, 62346244.

4 P. Botella, A. Corma and M. Quesada, J. Mater. Chem., 2012, 22, 6394-6401.

5 H. J. Zhang, Z. Y. Li, P. P. Xu, R. F. Wu and Z. Jiao, Chem. Commun., 2010, 46, 6783-6785.

6 P. Beyazkilic, A. Yildirim and M. Bayindir, Nanoscale, 2014, 6, 15203-15209.

7 Q. J. He, J. L. Shi, F. Chen, M. Zhu and L. X. Zhang, Biomaterials, 2010, 31, 3335-3346.

8 N. W. Clifford, K. S. Iyer and C. L. Raston, J. Mater. Chem., 2008, 18, 162-165.

9 X. Du and J. H. He, J. Colloid Interface Sci., 2010, 345, 269277.

10 I. Sokolov, Y. Y. Kievsky and J. M. Kaszpurenko, Small, 2007, 3, 419-423.

11 I. Sokolov and S. Naik, Small, 2008, 4, 934-939.

12 L. Tao, C. J. Song, Y. J. Sun, X. H. Li, Y. Y. Li, B. Q. Jin, Z. J. Zhang and K. Yang, Anal. Chim. Acta, 2013, 761, 194-200.

13 S. Palantavida, N. V. Guz and I. Sokolov, Part. Part. Syst. Charact., 2013, 30, 804-811.

14 S. Palantavida, N. V. Guz, C. D. Woodworth and I. Sokolov, J. Nanomed. Nanotechnol., 2013, 9, 1255-1262.

15 E. B. Cho, D. O. Volkov and I. Sokolov, Small, 2010, 6, 23142319.

16 S. P. Hudson, R. F. Padera, R. Langer and D. S. Kohane, Biomaterials, 2008, 29, 4045-4055.

17 Q. J. He, Z. W. Zhang, Y. Gao, J. L. Shi and Y. P. Li, Small, 2009, 5, 2722-2729.

18 X. Y. Zhang, X. Q. Zhang, S. Q. Wang, M. Y. Liu, Y. Zhang, L. Tao and Y. Wei, ACS Appl. Mater. Interfaces, 2013, 5, 1943-1947.

19 Q. J. He, Y. Gao, L. X. Zhang, Z. W. Zhang, F. Gao, X. F. Ji, Y. P. Li and J. L. Shi, Biomaterials, 2011, 32, 7711-7720.

20 Q. J. He, Y. Gao, L. X. Zhang, W. B. Bu, H. R. Chen, Y. P. Li and J. L. Shi, J. Mater. Chem., 2011, 21, 15190-15192. 
21 N. Vlachy, D. Touraud, J. Heilmann and W. Kunz, Colloids Surf., B, 2009, 70, 278-280.

22 T. L. Hwang, C. T. Sung, I. A. Aljuffali, Y. T. Chang and J. Y. Fang, Colloids Surf., B, 2014, 114, 334-341.

23 D. W. Loe and F. J. Sharom, Br. J. Cancer, 1993, 68, 342-351.

24 M. Liong, J. Lu, M. Kovochich, T. Xia, S. G. Ruehm, A. E. Nel, F. Tamanoi and J. I. Zink, ACS Nano, 2008, 2, 889-896.

25 L. T. Zhuravlev, Langmuir, 1987, 3, 316-318.

26 T. Yu, A. Malugin and H. Ghandehari, ACS Nano, 2011, 5, 5717-5728.

27 M. A. Rauf, J. P. Graham, S. B. Bukallah and M. A. S. Al-Saedi, Spectrochim. Acta, Part A, 2009, 72, 133-137.

28 T. Asefa and Z. Tao, Chem. Res. Toxicol., 2012, 25, 2265-2284.

29 C. Aielloa, P. Andreozzi, C. L. Mesa and G. Risuleo, Colloids Surf., B, 2010, 78, 149-154.

30 A. Yildirim, E. Ozgur and M. Bayindir, J. Mater. Chem. B, 2013, 1, 1909-1920.

31 Y. S. Lin and C. L. Haynes, J. Am. Chem. Soc., 2010, 132, 48344842.

32 D. Tarn, C. E. Ashley, M. Xue, E. C. Carnes, J. I. Zink and C. J. Brinker, Acc. Chem. Res., 2013, 46, 792-801.

33 E. Fröhlich, Int. J. Nanomed., 2012, 7, 5577-5591.
34 E. Gandin, Y. Lion and A. Vandevorst, Photochem. Photobiol., 1983, 37, 271-278.

35 T. Theodossiou, J. S. Hothersall, E. A. Woods, K. Okkenhaug, J. Jacobson and A. J. MacRobert, Cancer Res., 2003, 63, 18181821.

36 L. Xiao, L. Gu, S. B. Howell and M. J. Sailor, ACS Nano, 2011, 5, 3651-3659.

37 H. Maeda, J. Wu, T. Sawa, Y. Matsumura and K. Hori, J. Controlled Release, 2000, 65, 271-284.

38 H. Hillaireau and P. Couvreur, Cell. Mol. Life Sci., 2009, 66, 2873-2896.

39 J. Xie, C. J. Xu, N. Kohler, Y. L. Hou and S. H. Sun, Adv. Mater., 2007, 19, 3163-3166.

40 Q. Cai, Z. S. Luo, W. Q. Pang, Y. W. Fan, X. H. Chen and F. Z. Cui, Chem. Mater., 2001, 13, 258-263.

41 A. Yildirim, H. Budunoglu, B. Daglar, H. Deniz and M. Bayindir, ACS Appl. Mater. Interfaces, 2011, 3, 1804-1808.

42 A. Yildirim and M. Bayindir, J. Mater. Chem. A, 2015, 3, 38393846.

43 N. Lang and A. Tuel, Chem. Mater., 2004, 16, 1961-1966.

44 B. Garipcan, S. Odabas, G. Demirel, J. Burger, S. S. Nonnenmann, M. T. Coster, E. M. Gallo, B. Nabet, J. E. Spanier and E. Piskin, Adv. Eng. Mater., 2011, 13, B3-B9. 